\title{
Parent Flood Frequency Distribution of Turkish Rivers
}

\author{
Omer Levend Asikoglu* \\ Ege University, Department of Civil Engineering, Izmir, Turkey
}

Received: 4 April 2017

Accepted: 18 July 2017

\begin{abstract}
This study investigates the existence of a single parent flood frequency distribution on a Turkish scale. In the design of hydraulic structures, estimating the project flood of a given period or probability is usually the first step. Determining an acceptable design criterion depends substantially on the probability distribution of floods. In this study, annual peak series from 268 Turkish rivers was collected and a database of L-Moment ratios was constructed. The best-fit probability distribution (PD) model was investigated among seven distribution models (generalized logistic, GLO, generalized extreme value, GEV, generalized Pareto, GPA, three-parameter lognormal, LN3, Pearson type 3, P3, Gumbel, GUM, and normal distribution, N) by using a framework of L-moment ratio diagrams. The L-Moment ratio framework used in this study consisted of two sequential procedures. The first graphical procedure evaluated the L-Moment diagrams visually while the second procedure is based on the hypothetical testing procedure of L-Moment diagrams. The results of a graphical inspection of the dataset show GEV distribution as a potential parent PD of the floods in Turkey. A more detailed hypothetical testing procedure comprises Monte Carlo simulations produced from a GEV model. In a hypothetical testing procedure the variability of L-skewness and L-kurtosis values of sample data are situated within the theoretical limits of GEV distribution. Consequently, the GEV distribution is accepted as a single parent PD for annual maximum flow series of Turkey.
\end{abstract}

Keywords: parent flood frequency distribution, generalized extreme value distribution, Turkish floods, L-moment ratio diagram framework

\section{Introduction}

Determining the probability distribution (PD) of such random events as floods by estimating their behavior has always been a significant problem. Identifying the appropriate PD of floods is probably the most important step in flood frequency analysis. Accordingly, many studies all over the world have investigated how to determine an appropriate PD of floods [1-16]. The

*e-mail: omer.asikoglu@ege.edu.tr selection of techniques for assessing flood frequency in specific conditions depends on several dynamics, including national tradition, knowledge of the modeler, the aim of a study, governmental necessities, and data accessibility [17].

Cunnane [18] investigated the flood frequency determination techniques adopted by 55 organizations from 28 countries. Gumbel, two-parameter lognormal (LN2), P3, and log-Pearson type 3 (LP3) distributions appear to be the most common of the six extant distributions, with the other two being extreme value type 2 (EV2) and GEV distributions. 
McMahon et al. [19] examined the PD of yearly maximum flows of 974 gauging stations all over the world using conventional moment diagrams, and LP3 distribution provided a favorable fit to peak flows, while the LN2, GUM, and Gamma (GAM) distributions did not. Later studies show that the ordinary product moment diagrams generate false results because the estimators of conventional product moment ratios have remarkable bias, particularly for short and highly skewed records [20].

Onoz and Bayazit [3] investigated the suitability of seven PDs (N, LN2, LN3, GUM, GEV, P3, and LP3) to the observations of peak flows at 19 stations all over the world. The record lengths of data ranged from 60 years to 165 years. Using various procedures, they identified GEV as the "most appropriate distribution" in most cases. Therefore, GEV could be considered a universal PD for flood frequency analysis.

Vogel and Wilson [21] studied the selection of the PD function of yearly peak flows in the United States with data from 1,455 sites using L-moment diagrams. They identified the LP3, LN3, and GEV distributions as suitable PDs in the U.S., and considered other two- and three-parameter options as inappropriate for the whole continent.

According to Castellarin et al. [17], some European countries recommend GEV distribution (i.e., Austria, Italy, Germany, and Spain), while other countries prefer other frequently used distributions, such as GUM (i.e., Spain and Finland), and generalized Pareto distribution (GPA; i.e., Belgium). Moreover, in Slovenia the Agency of the Environment recommends the use of five distribution models (i.e., N, LN, P3, LP3, and GUM), Slovakia often uses the GAM, LN3, LP3, and GEV distributions, and the UK generally prefers the generalized logistic distribution (GLO) [15].

The PDs for flood frequency analysis are frequently selected by performing statistical tests, such as AndersonDarling, chi square, and Kolmogorov-Smirnov. Empirical appropriateness also plays a significant role in PD selection [18]. However, the aforementioned tests show some obvious restrictions, including non-objective and uncertain results, as reflected in the fact that more than one PD frequently passes the tests $[22,7]$.

A literature review identifies several criteria, such as Akaike information criterion $(A I C)$, Anderson-Darling criterion $(A D C)$, and Bayesian information criterion $(B I C)$ as significant alternatives to the above-mentioned statistical tests.

Laio et al. [7] showed that these criteria generated promising results in model selection, but their conclusions were not convincing enough because the criterion to be accepted in practical applications remains unknown. They stated that the AIC and BIC generally select the identical PD, while the ADC often selects a different PD.

The L-moments ratio diagrams, which are used to determine the observed data that are closest to the theoretical PD, are among the most common tools for selecting PDs in flood frequency studies [23]. However, this method is not completely objective since the goodness of fit of a theoretical PD to the sample data is usually determined based on graphical evaluation.

Pandey et al. [24] proposed the use of a distance-based measure between the observed and simulated L-kurtosis values to overcome the limitation of the graphical evaluation.

Likewise, Kroll and Vogel [25] proposed a criterion for addressing the difficulties in the graphical analysis of L-moment ratio plots. They also recommended the use of another distance-based measurement between the observed and simulated L-moment values [7].

In the last decade, considerable effort has been devoted to estimate the best-fit frequency distribution in flood frequency studies using the above-mentioned methods.

Saf [26] aimed to obtain flood frequency estimations from 47 sites in the western Mediterranean Basins of Turkey. Regional flood frequency estimations are made for three sub-regions using seven various distributions (GLO, GEV, N, P3, GPA, Wakeby, and Kappa). The L-moment goodness-of-fit statistic showed P3 as the most appropriate PD for Antalya and lower-western Mediterranean sub-regions, and GLO for the upperwestern Mediterranean sub-region.

Noto and La Loggia [27] analyzed annual maximum peak flow series from more than 50 gauged stations of Sicily in Italy. The region was divided into five subregions. Based on the L-moment ratio diagram and other statistical criteria, GEV was stated as the best-fit PD from various distributions. The flood frequency curves are constructed using GEV distribution.

Haddad and Rahman [11] examined BIC, AIC, AICsecond order variant (AICc), and a modified ADC with peak flow series from Tasmania in Australia. They found that $\mathrm{ADC}$ was more effective in identifying the parent $\mathrm{PD}$ properly than the AIC and BIC for three-parameter distributions. AIC and BIC, on the other hand, were better at representing the parent distribution for two-parameter distributions. From various frequency distributions, the two-parameter log normal distribution was found to be the best choice.

Rahman et al. [13] attempted to examine the suitability of 15 frequency distributions based on the peak flow series in Australia. The best-fit probability distribution was determined with the Anderson-Darling test, KolmogorovSmirnov test, BIC, and AIC. The L-moments ratio diagram was used to make a visual evaluation. They indicated that a single parent PD could not be identified for peak flows in all the states of Australia. However, the P3, GEV, and GPA distributions were determined as the best-fit distributions.

Salinas et al. [15] attempted to find a parent PD on a European scale by using a database for Europe that contained the yearly peak flows of 4,105 sites in 15 European countries. They also investigated how an appropriate PD could be selected using L-moment ratio diagrams. An initial examination of the database identified the GEV distribution as a potential PD for Europe. However, in a hypothesis-testing procedure, the 
Monte Carlo simulations rejected the use of the GEV as a single parent PD for Europe.

Kumar et al. [28] performed regional flood frequency analysis using the method of L-moments, artificial neural network (ANN), and fuzzy inference system (FIS) for the lower Godavari subzone of India with the annual peak flow data of 17 catchments. According to the L-moment ratio diagram and $Z_{\text {dist }}$ statistics, the $\mathrm{P} 3$ distribution was selected as the best-fit PD for the entire region.

Ahmad et al. [29] compared various parameter estimation methods to identify the best-fit probability distribution of annual peak flow series for flood frequency analysis in Pakistan. The best-fit probability distribution is determined using the probability plot correlation coefficient (PPCC) test, Anderson Darling (AD) test, and L-moments ratio diagram for each site. Their results indicate that GPA is the most appropriate PD for most of the stations, followed by GLO and GEV.

Koutsoyiannis [30] showed that the extreme value distribution of type II (EV2) is a consistent choice to apply to hydrological extremes. Based on the theoretical analyses, an extensive empirical investigation was performed using a collection of 169 of the longest available rainfall records worldwide, each having 100-154 years of data. The results verified the theoretical results.

This study constructed a dataset of L-moment ratios that comprised the annual peak flows of 268 Turkish rivers. The best-fit PD model was investigated using the L-moment ratio diagram framework. The most important advantage of the L-moment framework is that L-moment diagrams are evaluated not only visually but also as a measurable tool by investigating the appropriate probability distribution.

In the graphical inspection, the weighted moving average (WMA) series of L-coefficient of skewness and L-coefficient of kurtosis values ( $L-C s-L-C k$ pairs) were plotted on the L-moment ratio diagram. The visual inspection of WMA series identified the GEV model as a better candidate for annual maximum series compared with the other distributions (GLO, GPA, LN3, P3, GUM, and $\mathrm{N}$ ). A more detailed hypothetical testing procedure - including 10,000 Monte Carlo simulations produced from a GEV model - reveals that GEV distribution successfully characterizes the Turkish floods as a single parent frequency distribution.

\section{Data and Study Area}

This work examines the annual flood peak datasets of 268 river gauging stations all over Turkey. The record lengths range between 25 and 65 years with a mean record length of 35 years. Fig. 1 shows the physical locations of these stations. The gauging stations are scattered all over the country rather uniformly.

Turkey is located in the northern hemisphere on the Anatolian Plateau and the Thrace Peninsula, in an eastwest direction between Europe and Asia. The territory of Turkey lies between $36-42^{\circ}$ north parallels and $26-45^{\circ}$ east meridians, and roughly resembles a rectangle. The country is surrounded by the Mediterranean Sea, the Black Sea, and the Aegean Sea. There are generally four climate types in the country. In the north, the Black Sea climate is rainy in all seasons; in the west and south, the Mediterranean climate is hot and dry in summers and warm and rainy in winters; in Central Anatolia and in Southeastern Anatolia a semi-arid climate exists, and in Eastern Anatolia the continental climate is very cold in winter and more or less rainy in the long summers.

\section{L-moment Ratio Diagram Framework \\ L-moments and L-moment Ratio Diagrams}

L-moments are linear combinations of order statistics, which are robust to outliers and almost unbiased for short data series. These advantages make L-moments especially appropriate for flood frequency analysis, as well as determining probability distribution and estimation of distribution parameters [23]. Greenwood et al. [31] defined L-moments as linear combinations of probability-weighted moments (PWM):

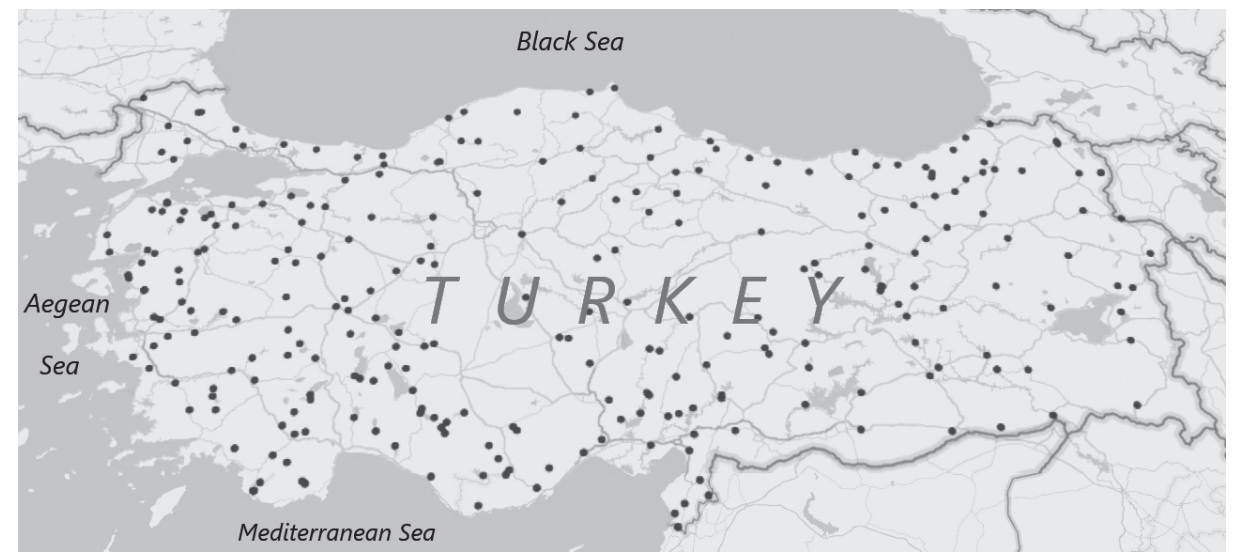

Fig. 1. The locations of flow-gauging stations. 


$$
\beta_{r}=E\left\{X[F(x)]^{r}\right\}
$$

...where $F(x)$ is the underlying probability distribution function of $x$. The L-moments are given as the linear combinations of PWMs:

$$
\begin{gathered}
\lambda_{1}=\beta_{0} \\
\lambda_{2}=2 \beta_{1}-\beta_{0} \\
\lambda_{3}=6 \beta_{2}-6 \beta_{1}-\beta_{0} \\
\lambda_{4}=20 \beta_{3}-30 \beta_{2}+12 \beta_{1}-\beta_{0}
\end{gathered}
$$

$\lambda_{1}$ and $\lambda_{2}$ are the L-mean and L-standard deviation and determine central tendency and dispersion, respectively. The L-Moment ratios are calculated as follows:

$$
\begin{aligned}
& L C v=\lambda_{2} / \lambda_{1} \\
& L C s=\lambda_{3} / \lambda_{2} \\
& L C k=\lambda_{4} / \lambda_{2}
\end{aligned}
$$

Hosking [23] showed that the absolute value of $L-C s$ and $L-C k$ are smaller then $1(|L-C s|<1,|L-C k|<1)$ and that the $L-C v$ is in the range of $0<L-C v<1$, for $x \geq 0$.

The L-moment ratio diagrams assess the fitness of different PDs by inspecting the closeness of the sample L-moments to the curves of different theoretical PDs [23]. The importance of L-moment ratio diagrams in selecting an appropriate PD has been frequently cited in the literature [23-24, 26-29, 32-37]. A further advantage of these diagrams is the convenience of L-moments, particularly for short data since the L-moment estimates are less biased than the product moment estimates.

In practice, the L-moment ratio diagrams take two different forms. The first diagram $(L-C v-L-C s)$ includes plots of the L-coefficient of variation versus the L-coefficient of skewness and is used for two-parameter PDs, while the second diagram $(L-C s-L-C k)$ plots the L-coefficient of skewness against the L-coefficient of kurtosis and is frequently used in studying threeparameter PDs. This study employs the $L-C s-L-C k$ diagram that compares the considered three-parameter distributions, and also N and GUM together.

\section{Graphical Inspection Using Moving Average Series}

Empirical data usually include noise because of sampling uncertainty. Salinas et al. [15] proposed the use of WMA series (moving average series weighted with record length) to reduce noise and effectively examine the most appropriate PD that reflects the statistical characteristics of the observed data. The WMA is obtained by calculating the weighted average of 10 adjacent $L-C s$ samples and plotting them against the weighted average of the 10 corresponding $L-C k$ samples on an L-moment ratio diagram. Therefore, to decrease the effect of sampling variability in small samples, each L-moment ratio is proportionally weighted to the sample record length [15].

Fig. 2 shows the L-moment ratio diagram that includes the L-moment ratios of all flow stations (gray circles) as well as five lines and two plots that illustrate the theoretical $L-C s-L-C k$ relationship of the PDs, including GEV, GLO GPA, LN3, PE3, N, and GUM. The WMA line, which represents the average behavior of sample L-moment ratios, appears closer to the theoretical distribution curve of GEV than the other PDs. The trend of the WMA line shows that GEV can better represent the frequency characteristics of the annual peak flows of the Turkish rivers than the other considered PDs.

\section{Hypothetical Testing Procedure}

A graphical analysis using the moving average series shows that GEV distribution best represents the mean statistical properties of all data among all available PDs. To test whether the GEV distribution is an effective parent PD of related peak flows, Salinas et al. [15] proposed a comparison of the dispersion of sample L-moment ratios and the random L-moment ratios that were produced from the GEV distribution via Monte Carlo simulations.

Given that the sample distribution passed the normality test, the $L-C s$ rates of the peak flow series are expected to be random across the stations as defined with a normal distribution with a mean of 0.25 and a standard deviation of 0.13. In the Monte Carlo simulations, 10,000 $L-C s$ values are generated from the normal distribution. Using these values, 10,000 stations are produced from a GEV distribution with a mean data length of 35 years.

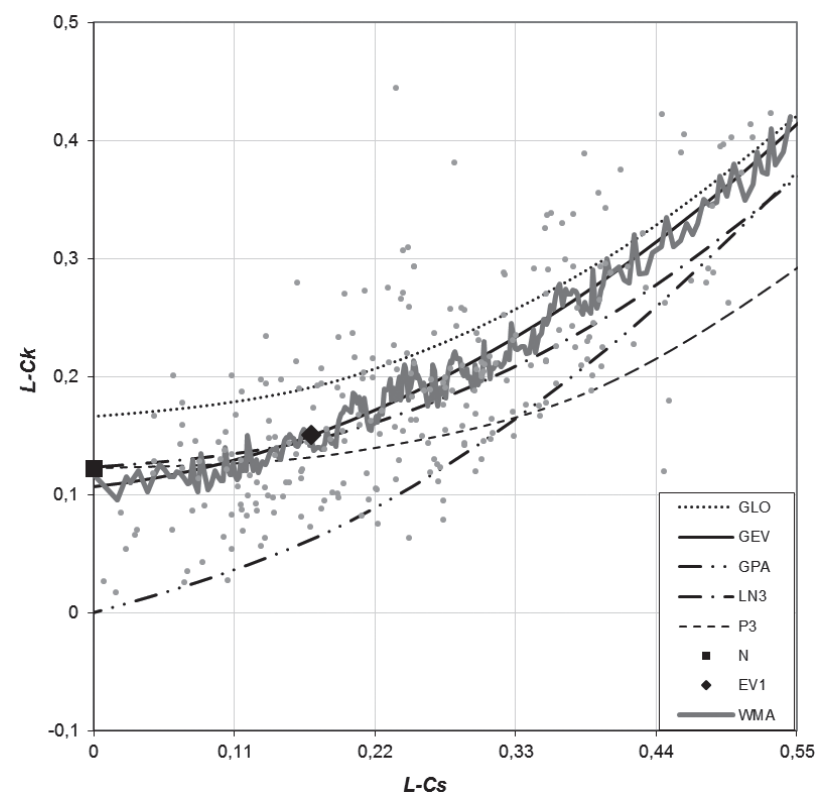

Fig. 2. $L-C s-L-C k$ diagram with the weighted moving average (WMA) series for the whole database. 


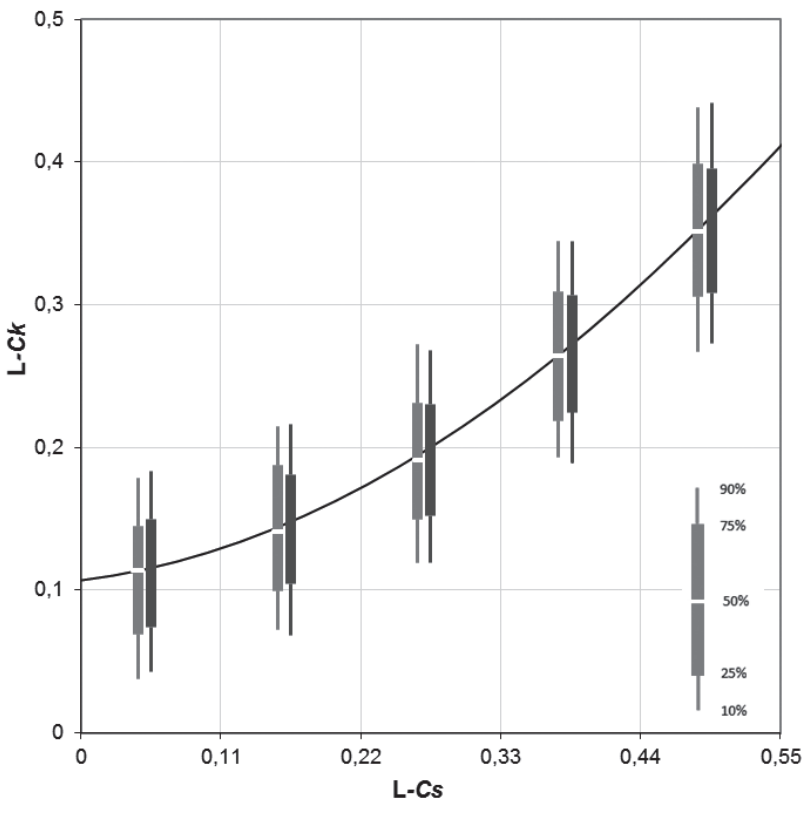

Fig. 3. The distribution of $10,000 L-C k$ simulations, all randomly drawn from GEV distribution (blue box plots) and the distribution of sample $L-C k$ values (red box plots).

The L-moments and their ratios are calculated from the peak flow data of the 10,000 generated GEV stations. In the $L-C s-L-C k$ diagram, these $10,000 L-C s-L-C k$ would not necessarily be located on the theoretic curve of the GEV distribution because of the biases in the estimations of L-moments [15].

To simplify the analysis, the diagram is split into five bins with equal distances for $L-C s$ values ranging from 0 to 0.55 . The dispersion of the $L-C k$ rates for each bin is signified by the $10,25,50,75$, and $90 \%$ percentiles. Fig. 3 presents the related box plots and the GEV curve.

A statistical hypothesis testing procedure is performed to test whether the PD of all Turkish stations represented by the GEV model is to be accepted or rejected. The null hypothesis $\mathrm{H}_{0}$ posits that for each bin, the $p$ th percentile of the $L-C k$ values of the observed data does not significantly differ from the $p$ th percentile of all stations that are randomly drawn from the GEV distribution.

The percentiles are distributed according to binomial distribution. And for the series with a sample length of $n>20$, the binomial distribution approximates a normal distribution with a mean of $n p$ and a standard deviation of $\sqrt{n p(1-p)}$. Conover [38] proposed calculating the confidence intervals of the percentiles according to the rank number in the ordered data. The lower and upper confidence limits are calculated as follows and then rounded up to the next integer:

$$
\begin{aligned}
& l=n p-z_{\propto / 2} \sqrt{n p(1-p)} \text { and } \\
& u=n p+z_{\propto / 2} \sqrt{n p(1-p)}
\end{aligned}
$$

...where $z$ is the standard normal deviate. The $l$ th and $u$ th observations denote the lower and upper confidence limits of the related percentile with a confidence level of $(1-\alpha) \%$.

Table 1 shows the observed $L-C k$ percentiles in each bin. At the $5 \%$ significance test, all of the observed $L-C k$ percentiles are within the confidence limits, whereas the dispersions of the $L-C k$ percentiles of the observed and simulated data do not significantly differ from each other in any of the L-Cs bins. At the $10 \%$ significance test, only one value of the $L-C k$ percentiles was outside the confidence limits (in bold). The null hypothesis, which posits that the percentiles $\left(L-C k_{10}, L-C k_{25} L-C k_{50}, L-C k_{75}\right.$, and $L-C k_{90}$ ) of the sample data are equal to the percentiles of the randomly generated GEV series of Turkish floods, is accepted for all the percentiles and bins with a significance level of $\alpha=5 \%$, as well as for almost all the percentiles and bins with a significance level of $\alpha=10 \%$.

The result of the hypothetical testing procedure is more robust than that of the graphical inspection of the weighted moving average series.

\section{Discussion}

The results of both the graphical inspection and hypothetical testing procedures using the Monte Carlo simulations indicate that choosing GEV as a parent PD of Turkish floods fully describes the variability of sample L-moment ratios. The graphical inspection hints about the suitability of GEV distribution. The simulation results concerning the dispersion of L-moment ratios around the

Table 1. The observed $L-C k$ values of percentiles (The value rejecting a $10 \%$ significance test shown in bold).

\begin{tabular}{|c|c|c|c|c|c|}
\hline \multirow{2}{*}{$L$ - $C k$ percentile } & \multicolumn{5}{|c|}{ Range of $L$-Cs values per bin } \\
\cline { 2 - 6 } & $0 .-0.11$ & $0.11-0.22$ & $0.22-0.33$ & $0.33-0.44$ & $0.44-0.55$ \\
\hline $90 \%$ & 0.178 & 0.215 & 0.272 & 0.344 & 0.438 \\
\hline $75 \%$ & 0.145 & 0.187 & 0.231 & 0.309 & 0.399 \\
\hline $50 \%$ & 0.114 & 0.141 & 0.191 & 0.264 & 0.351 \\
\hline $25 \%$ & 0.069 & 0.099 & 0.150 & 0.219 & 0.306 \\
\hline $10 \%$ & 0.038 & 0.072 & 0.119 & 0.193 & $\mathbf{0 . 2 6 7}$ \\
\hline
\end{tabular}


GEV distribution curve indicate that the spread of $L-C k$ rates is within the accepted confidence limits. Therefore, GEV is an appropriate parent PD for representing the behavior of floods with regard to the sample L-moment ratios.

As the main inspiration for this study, Salinas et al. [15] showed that the GEV distribution alone could not perfectly define the variability of the L-moments estimated from the European database. They emphasized that a single analytical expression on large scales and in different processes could not sufficiently explain all possible local characteristics. However, they identified the GEV distribution as an acceptable parent PD for representing the median behavior of the L-moment ratios of the observations.

\section{Conclusions}

Several methods in the literature can be used to determine the most appropriate PD model for floods. As described in the introduction, statistical tests (i.e., Kolmogorov-Smirnov, chi-square, and AndersonDarling tests), criteria (i.e., AIC, BIC, and ADC), and graphical tests (i.e., product moment ratio and L-moment ratio diagrams) are the most commonly used techniques for PD model selection. However, each of these methods has some incomplete or objectionable aspects. The L-moment framework used in this study demonstrate that with the help of measurable criteria, the evaluations from the L-moment ratio diagrams are more powerful than those from visual analysis.

This study examines a single parent probability distribution of Turkish floods across different locations and scales. A graphical inspection using moving average series and a hypothetical testing procedure using Monte Carlo simulations are conducted one after another. Through a graphical inspection, the WMA line shows that the GEV distribution can better represent the frequency of the annual peak flows of Turkish rivers compared with the other PDs. A hypothetical inspection is also performed by comparing the dispersion of L-moment ratios of the observed data and the simulations where each stations represents a random sample of L-moment ratios that are produced from GEV distributions. The dispersion of the $L-C k$ rates for each bin is signified by the $10,25,50,75$, and $90 \%$ percentiles and presented with the related box plots and the GEV curve in Fig. 3. The null hypothesis, which posits that the $L-C k$ percentiles of the sample data are equal to the $L-C k$ percentiles from a randomly generated GEV series of Turkish floods, is accepted for all cases with a significance level of $\alpha=5 \%$ and for almost all cases with a significance level of $\alpha=10 \%$. In conclusion, the GEV distribution, as a single parent PD, successfully represents the statistical properties of Turkish floods.

\section{References}

1. CHOWDHURY J., STEDINGER J., LU L. Goodnessof-fit tests for regional generalized extreme value flood distributions, Water Resources Research, 27, 1991.

2. BOBEE B., CAVADIAS G., ASHKAR F., BERNIER J., RASMUSSEN P. Towards a systematic approach to comparing distributions used in flood frequency analysis. Journal of Hydrology, 142, 121, 1993.

3. ONOZ B., BAYAZIT M. Best-fit Distributions of Largest Available Flood Samples, Journal of Hydrology, $167,1995$.

4. MITOSEK H.T, STRUPCZEWSKI W.G., SINGH V.P Three procedures for selection of annual flood peak distribution, Journal of Hydrology, 323, 2006.

5. MERZ R., BLOSCHL G., HUMER G. National flood discharge mapping in Austria, Natural Hazards, 46, 2008.

6. DI BALDASSARE G., LAIO F., MONTANARI A. Design flood estimation using model selection criteria Physics and Chemistry of the Earth, Parts A/B/C 34 (10-12), 2009.

7. LAIO F., DI BALDASSARE G., MONTANARI A. Model selection techniques for the frequency analysis of hydrological extremes. Water Resources Research, 45, W07416, 2009.

8. CALENDA G., MANCINI C.P., VOLPI E. Selection of the probabilistic model of extreme floods: The case of the River Tiber in Rome. Journal of Hydrology. 371, 1, 2009.

9. NOTO L.V., LA LOGGIA G. Use of L-moments approach for regional flood frequency analysis in Sicily, Italy. Water resources management, 23, 2207, 2009.

10. GAMAGE W., S.H.P., HEWA G.A., SUBHASHINI W.H.C., DANIELL T.M., KEMP D. Modelling the extreme floods of South Australian catchments, MODSIM, 3435, 2009.

11. HADDAD K., RAHMAN A. Selection of the best fit flood frequency distribution and parameter estimation procedure: a case study for Tasmania in Australia. Stoch. Env. Res. Risk A. 25, 415, 2011.

12. AHMAD U.N., SHABRI A., ZAKARIA Z.A. Flood frequency analysis of annual maximum stream flows using L-moments and TL-moments approach. Applied Mathematical Sciences. 5, 243, 2011.

13. RAHMAN A.S., RAHMAN A., ZAMAN M.A., HADDAD K., AHSAN A., IMTEAZ M. A study on selection of probability distributions for at-site flood frequency analysis in Australia, Natural Hazards, 69 (3), 2013.

14. SALARPOUR M., YUSOP Z., YUSOF F. Comparison of Distribution Models for Peak flow, Flood Volume and Flood Duration. Research Journal of Applied Sciences, Engineering and Technology, 6, 733, 2013

15. SALINAS J.L., CASTELLARIN A., KOHNOVA S., KJELDSEN T.R. Regional parent flood frequency distributions in Europe - Part 1: Is the GEV model suitable as a pan-European parent? Hydrology and Earth System Sciences, 18, 2014

16. KUMAR R., GOEL N.K., CHATTERJEE C., NAYAK P.C. Regional Flood Frequency Analysis using Soft Computing Techniques. Water Resour. Manag. 29, 1965, 2015.

17. CASTELLARIN A., KOHNOVA S., GAAL L., FLEIG, A., SALINAS J.L., TOUMAZIS A., KJELDSEN T.R., MACDONALD N. Review of applied-statistical methods for flood-frequency analysis in Europe. NERC/Centre 
for Ecology and Hydrology, 122 (ESSEM COST Action ES0901), 2012.

18. CUNNANE C. Statistical distributions for flood frequency analysis. WMO Operational Hydrology Report no. 33, WMO No. 718, WMO Secretariat, Geneva, Switzerland. 1989.

19. McMAHON T.A., FINLAYSON B.L. HAINES A.T., SRIKANTHAN R. Global Runoff: Continental Comparisons of Annual Flows and Peak Discharges. Catena Verlag, Cremlingen-Germany. 1992.

20. VOGEL R.M., FENNESSEY, N.M. L-moment diagrams should replace product moment diagrams, Water Resources Research, 29, 6, 1993.

21. VOGEL R. M., WILSON I. Probability distribution of annual maximum, mean, and minimum stream flows in the United States. Journal of Hydrological Engineering. 1 (2), 1996

22. BURNHAM K.P., ANDERSON D.R. Model Selection and Multimodel Inference, 2nd ed., Springer, New York, 2002.

23. HOSKING J.R.M. WALLIS J.R. Regional Frequency Analysis: An Approach Based on L-moments. Cambridge University Press. 1997.

24. PANDEY M.D., VAN GELDER P., VRIJLING K. Assessment of an L-kurtosis-based criterion for quantile estimation, Journal of Hydrologic Engineering, 6 (4), 2001.

25. KROLL C.N., VOGEL R.M. Probability distribution of low streamflow series in the United States, Journal of Hydrologic Engineering, 7 (2), 2002.

26. SAF B. Regional Flood Frequency Analysis Using L-Moments for the West Mediterranean Region of Turkey. Water Resour. Manag. 23, 531, 2009.

27. NOTO L.V., LA LOGGIA G. Use of L-Moments Approach for Regional Flood Frequency Analysis in Sicily, Italy. Water Resour. Manag. 23, 2207, 2009

28. KUMAR R., GOEL N.K., CHATTERJEE C., NAYAK P.C. Regional Flood Frequency Analysis using Soft Computing Techniques. Water Resour. Manag. 29, 1965, 2015.
29. AHMAD I., FAWAD M., AKBAR M., ABBAS A., ZAFAR, H. Regional Frequency Analysis of Annual Peak Flows in Pakistan Using Linear Combination of Order Statistics Pol. J. Environ. Stud. 25, 6, 2016.

30. KOUTSOYIANNIS D. Statistics of extremes and estimation of extreme rainfall: II. Empirical investigation of long rainfall records, Hydrological Sciences Journal, 49, 4, 2004.

31. GREENWOOD J.A., LANDWEHR J.M., MATALAS N.C., WALLIS J.R. Probability weighted moments: definition and relation to parameters of several distributions expressible in inverse form. Water Resources Research. 15, 1049, 1979.

32. DEKA S., BORAH M., KAKATY S.C. Distribution of Annual Maximum Rainfall Series of North-East India. European Water (EW) Publications 27, 3, 2009.

33. SHABRI A., ARIFF N. Frequency analysis of maximum daily rainfalls via 1-moment approach. Sains Malaysiana, 38, 149, 2009

34. AHMAD U.N., SHABRI A., ZAKARIA Z.A. Flood frequency analysis of annual maximum stream flows using Lmoments and TL-moments approach. Applied Mathematical Sciences. 5, 243, 2011.

35. IZINYON O.C., EHIOROBO J.O. L-moments approach for flood frequency analysis of river Okhuwan in Benin- Owena River basin in Nigeria. Nigerian Journal of Technology. 33, 10, 2014

36. STRUPCZEWSKI W.G., KOCHANEK K., MARKIEWICZ I., BOGDANOWICZ E., WEGLARCZYK S., SINHG V.P. On the tails of distributions of annual peak flow, Hydrology Research, 42, 171, 2011

37. AHMAD I., ABBAS A., SAGHIR A., FAWAD M. Finding Probability Distributions for Annual Daily Maximum Rainfall in Pakistan Using Linear Moments and Variants Pol. J. Environ. Stud. 25, 3, 2016.

38. CONOVER W.J. Practical Nonparametric Statistics, Third Edition, New York: John Wiley \& Sons. 1999. 